\title{
A SIMPLE METHOD FOR THE SOLUTION OF THE STELLAR STRUCTURE EQUATIONS INCLUDING ROTATION AND TIDAL FORCES
}

\author{
R. KIPPENHAHN and H.-C. THOMAS \\ Universitätssternwarte Göttingen and Max-Planck-Institut für Physik und \\ Astrophysik, München, Germany
}

\begin{abstract}
In the following a method is presented for computing the internal structure of nonspherical stars assuming that the force per gram causing the deviation from spherical symmetry is conservative. The method has the advantage that in a normal (spherical) stellar structure code only slight changes have to be made in order to obtain nonspherical stellar models. The method can be applied as well to rotating stars as to stars distorted by tidal effects. Although it is similar to that of Faulkner et al. (1968) in the case of purely rotating stars, it is not necessary to use the division into two zones, where either slow rotation or negligible contribution to the gravitional potential is assumed.
\end{abstract}

\section{Mathematical Definitions}

We consider a potential function $\Psi(x, y, z)$. The equipotential surfaces then are defined by $\Psi=$ const. We assume them to be topologically equivalent to spheres. The volume enclosed by a surface $\Psi=$ const. may be $V_{\Psi}$, the surface area $S_{\Psi}$.

For any function $f(x, y, z)$ we define the mean value over an equipotential surface $\Psi=$ const. by

$$
f=\frac{1}{S_{\Psi}} \int_{\Psi=\text { const. }} f \mathrm{~d} \sigma
$$

where $\mathrm{d} \sigma$ is the surface element of the surface $\Psi=$ const. By definition

$$
S_{\Psi}=\int_{\Psi=\text { const. }} \mathrm{d} \sigma
$$

The mean value $\bar{f}$ can be determined for each equipotential; it therefore is a function of $\Psi$ only, $\bar{f}=\bar{f}(\Psi)$. The distance $\mathrm{d} n$ between two neighboring equipotential surfaces $\Psi=$ const. and $\Psi+\mathrm{d} \Psi=$ const. is in general not constant. We define a function $g(x, y, z)$ by

$$
g=\mathrm{d} \Psi / \mathrm{d} n .
$$

From the function $g$ the mean values $\bar{g}$ and $\overline{g^{-1}}$, both being functions of $\Psi$, can be determined. One then gets for the volume $\mathrm{d} V_{\Psi}$ between the surfaces $\Psi$ and $\Psi+\mathrm{d} \Psi$

$$
\mathrm{d} V_{\Psi}=\int_{\Psi=\text { const. }} \mathrm{d} n \mathrm{~d} \sigma=\mathrm{d} \Psi \int_{\Psi=\text { const. }} \frac{\mathrm{d} n}{\mathrm{~d} \Psi} \mathrm{d} \sigma=\overline{g^{-1}} S_{\Psi} \mathrm{d} \Psi .
$$

In analogy to the sphere we define a 'radius' $r_{\Psi}$ by

$$
V_{\Psi}=\frac{4 \pi}{3} r_{\Psi}^{3}
$$


The new quantity $r_{\Psi}$ is a function of $\Psi$. The surface area $S_{\Psi}$ in general is not equal to $4 \pi r_{\Psi}^{2}$; we therefore define a new quantity $u$ by

$$
u=S_{\Psi} / 4 \pi r_{\Psi}^{2}
$$

where again $u$ is a function of $\Psi$ only, $u=u(\Psi)$. If the surfaces $\Psi=$ const. are spheres, $u$ is equal to 1 . We define by $M_{\Psi}$ the mass enclosed by the surface $\Psi=$ const. If $\Psi$ defines the mechanical potential of a vector field $\mathbf{g}$ :

$$
\mathbf{g}=-\nabla \Psi
$$

we can define the two functions

$$
v=\bar{g} \frac{r_{\Psi}^{2}}{G M_{\Psi}}, \quad w=\overline{g^{-1}} \frac{G M_{\Psi}}{r_{\Psi}^{2}},
$$

where $G$ is the gravitational constant. If the function $\Psi$ is the gravitational potential of a selfgravitating sphere the surfaces $\Psi=$ const. are spheres $(u=1)$, and

$$
g=G M_{\Psi} / r_{\Psi}^{2}
$$

is constant on these spheres and therefore $v$ and $w$ are constant and equal to 1 .

The Equations (1)-(8) are pure mathematical definitions. We will apply them to gravitational potential fields distorted by perturbing forces. It is worth mentioning that the Equations (1)-(8) do not contain any simplifying assumptions.

\section{Stellar Structure Equations}

A star may be distorted by centrifugal or tidal forces. The perturbing acceleration may be conservative. The potential of the total (i.e. gravitational plus perturbing) acceleration may be $\Psi$. In the following we assume the function $\Psi(x, y, z)$ to be known. We then derive the stellar structure equations for nonspherical stars, the nonsphericity being defined by the nonspherical equipotential surfaces. We start with spherical stars and use the well-known fact that $P, \varrho$ in hydrostatic equilibrium are constant on equipotentials. The mass $\mathrm{d}_{\Psi}$ between two equipotentials $\Psi$ and $\Psi+\mathrm{d} \Psi$ is according to (5)

$$
\mathrm{d} M_{\Psi}=\varrho(\Psi) \mathrm{d} V_{\Psi}=4 \pi r_{\Psi}^{2} \varrho(\Psi) \mathrm{d} r_{\Psi} .
$$

We therefore get

$$
\mathrm{d} r_{\Psi} / \mathrm{d} M_{\Psi}=1 / 4 \pi r_{\Psi}^{2} \varrho .
$$

From (4), (10) we get

$$
\mathrm{d} \Psi=\left(\frac{\mathrm{d} V_{\Psi}}{\mathrm{d} \Psi}\right)^{-1} \mathrm{~d} V_{\Psi}=\left(\frac{\mathrm{d} V_{\Psi}}{\mathrm{d} \Psi}\right)^{-1} \frac{\mathrm{d} M_{\Psi}}{\varrho}=\frac{\mathrm{d} M_{\Psi}}{\overline{g^{-1}} S_{\Psi} \varrho}
$$

and with (6), (8)

$$
\mathrm{d} \Psi=\frac{G M_{\Psi} \mathrm{d} M_{\Psi}}{\varrho u w 4 \pi r_{\Psi}^{4}} .
$$


The condition for hydrostatic equilibrium, $\mathrm{d} P / \mathrm{d} \Psi=-\varrho$, can now be written with (13) in the form

$$
\frac{\mathrm{d} P}{\mathrm{~d} M_{\Psi}}=-\frac{G M_{\Psi}}{4 \pi r_{\Psi}^{4}} f_{P},
$$

where

$$
f_{P}=\frac{1}{u w}=\frac{4 \pi r_{\Psi}^{4}}{G M_{\Psi} S_{\Psi}} \frac{1}{\overline{g^{-1}}} .
$$

The factor $f_{P}$ is a function of $\Psi$. If $\Psi$ is known the equipotential surfaces can be determined, and with them $S_{\Psi}, r_{\Psi}, \bar{g}$, and $\overline{g^{-1}}$ for each surface simply from the geometry of the equipotentials. The mass $M_{\Psi}$ depends on the density distribution $\varrho(\Psi)$ and in principle can be determined from (10) by integration.

If $L_{\Psi}$ is the energy which passes per second through the equipotential $\Psi=$ const. in the outward direction, then the increase $\mathrm{d} L_{\Psi}$ of $L_{\Psi}$ between the equipotential surfaces $\Psi$ and $\Psi+\mathrm{d} \Psi$ is given by

$$
\mathrm{d} L_{\Psi}=\varepsilon \mathrm{d} M_{\Psi}
$$

where $\varepsilon$ is the nuclear energy generation per gram per sec. For chemically homogeneous stars $\varepsilon$ depends only on $\varrho$ and $T$ and is therefore constant on equipotential surfaces

$$
\mathrm{d} L_{\Psi} / \mathrm{d} M_{\Psi}=\varepsilon .
$$

In these energy equations we neglected an additional term which contains the time derivative of the entropy. This may be permitted as long as we consider variations in time which are slow compared to the Kelvin-Helmholtz time scale.

For the case of energy transport by radiation we have

$$
F=-\frac{4 a c T^{3} \mathrm{~d} T}{3 \kappa \varrho} \frac{4 a c T^{3}}{\mathrm{~d} n} g \frac{\mathrm{d} T}{3 \kappa \varrho} .
$$

This equation contains the well-known fact that the radiative fiux $F$ varies on an equipotential surface proportionally to $g$. With Equation (13) we obtain

$$
F \cdot=-\frac{4 a c T^{3}}{3 \kappa} g \underset{\mathrm{d} M_{\Psi}}{\mathrm{d} T} \frac{u w \cdot 4 \pi r_{\Psi}^{4}}{G M_{\Psi}}
$$

In order to get $L_{\Psi}$ we integrate Equation (19) over the equipotential $\Psi=$ const. and obtain

and

$$
L_{\Psi}=\int_{\Psi=\text { const. }} F \mathrm{~d} \sigma=-\frac{16 \pi a c T^{3}}{3 \kappa} \bar{g} S_{\Psi} \mathrm{d} T M_{\Psi} \frac{u w r_{\psi}^{4}}{G M_{\Psi}}
$$

$$
L_{\Psi}=-\frac{64 \pi^{2} a c T^{3} r_{\Psi}^{4}}{3 \kappa} u^{2} v w \frac{\mathrm{d} T}{\mathrm{~d} M_{\Psi}},
$$


or

with

$$
\frac{\mathrm{d} T}{\mathrm{~d} M_{\Psi}}=-\frac{3 \kappa L_{\Psi}}{64 \pi^{2} a c T^{3} r_{\Psi}^{4}} f_{T},
$$

$$
f_{T}=\frac{1}{u^{2} v w}=\left(\frac{4 \pi r_{\Psi}^{2}}{S_{\Psi}}\right)^{2} \frac{1}{\bar{g}} \frac{1}{g^{-1}} .
$$

Equations (11), (14), (17), (22) become the four well-known stellar structure equations for the case of spherical configurations, for which $f_{P}=f_{T}=1$. In the nonspherical case we assume that $f_{P}, f_{T}$ are known as functions of $M_{\Psi}$. We can then solve the stellar structure equations. But in order to get a consistent solution one has to check, whether the equipotential surfaces and the functions $f_{P}, f_{T}$ are consistent with this model. For instance, that part $\phi$ of $\Psi$, which corresponds to the self-gravitation, must satisfy the Poisson equation $\Delta \phi=4 \pi G \varrho$.

\section{The Uniformly Rotating Star}

In order to solve the stellar structure equations one has to know the correction factors $f_{\boldsymbol{P}}$ and $f_{T}$ as functions of $r_{\psi}$. They are pure geometrical quantities, depending only on the form of the equipotential surfaces. In principle it is necessary to solve the Poisson equation simultaneously with the stellar structure equations. In our method we approximate the potential simply by the dimensionless expression (the 'geometry function')

$$
\tilde{\Psi}=\frac{1}{x}+\frac{1}{2} x^{2}\left(1-\mu^{2}\right)
$$

with

$$
x=\left(\omega^{2} / G M\right)^{1 / 3} r, \quad \mu=\cos \vartheta .
$$

We also redefine $v$ and $w$ as

with

$$
v=\overline{(\mathrm{d} \widetilde{\Psi} / \mathrm{d} n)} /\left(1 / y^{2}\right), \quad w=\overline{(\overline{\mathrm{d}} \widetilde{\Psi} / \mathrm{d} n)^{-1}} / y^{2}
$$

$$
y=\left(\omega^{2} / G M\right)^{1 / 3} r_{\Psi}
$$

instead of using Equations (8). Although in Equations (8) $M_{\Psi}$ and $r_{\Psi}$ appear simultaneously, the mass $M_{\Psi}$ does not appear any more in the definition of $v$ and $w$. The reason is that the geometry function is only used to describe the deviations from sphericity of the equipotentials.

Although our $\widetilde{\Psi}$ is identical with the normalized Roche potential the models computed by the method described are realistic stellar models with finite central density and not Roche models. This can immediately be seen if one applies the method to the limiting case of a nonrotating star. The equipotential surfaces are spheres; therefore $f_{P}=f_{T}=1$ and one gets the normal stellar structure equations and not spherical Roche models. The fact that stellar models are regular in the center although a geometry function is used which is singular there is a major advantage of our method. 


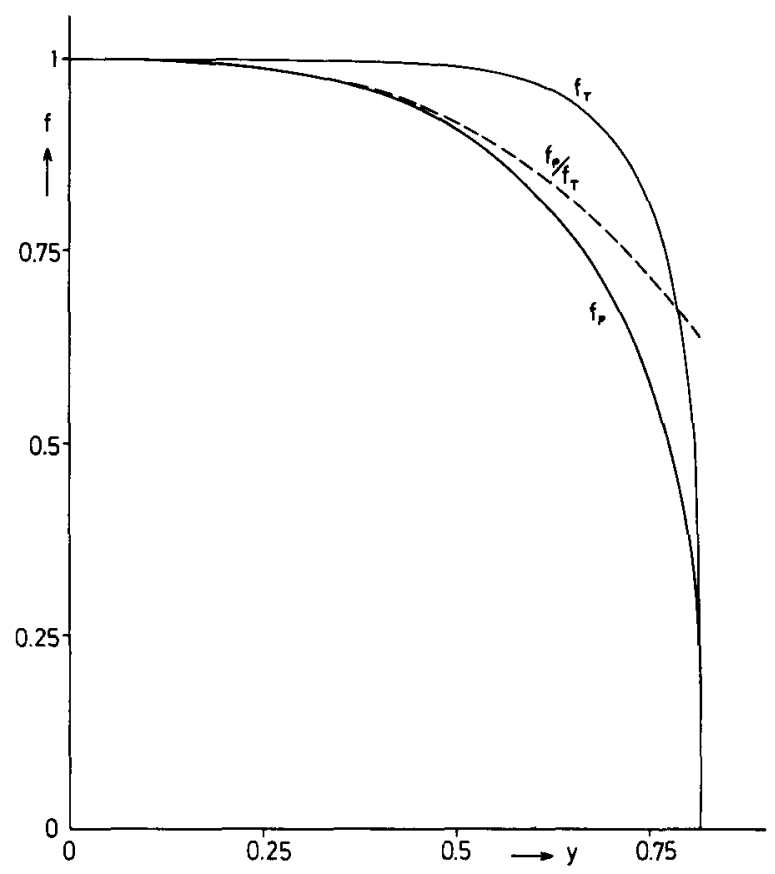

Fig. 1. The correction factors $f_{P}$ and $f_{T}$ as functions of $y=\left(\omega^{2} / G M\right)^{1 / 3} r \Psi$ for the case of pure solid body rotation. The ratio of the two functions is plotted in order to show the behavior near the surface.

Once the function $\Psi$ is specified and

$$
\begin{aligned}
& \widetilde{\Psi}=\text { const. } \\
& V_{\Psi}=\int_{0} \mathrm{~d} \tau, \\
& S_{\Psi}=\int_{\tilde{\Psi}=\text { const. }} \mathrm{d} \sigma, \\
& \frac{\overline{\mathrm{d} \widetilde{\Psi}}}{\mathrm{d} n}=\frac{1}{S_{\Psi}} \int_{\widetilde{\Psi}=\text { const. }} \frac{\mathrm{d} \tilde{\Psi}}{\mathrm{d} n} \mathrm{~d} \sigma, \\
& \overline{\left(\frac{\mathrm{d} \tilde{\Psi}}{\mathrm{d} n}\right)^{-1}}=\frac{1}{S_{\Psi}} \int_{\Psi=\text { const. }}\left(\frac{\mathrm{d} \tilde{\Psi}}{\mathrm{d} n}\right)^{-1} \mathrm{~d} \sigma
\end{aligned}
$$

are computed, $f_{P}$ and $f_{T}$ can be determined (Figure 1).

In the case of a corotating star in a close binary system

$$
\tilde{\Psi}=\frac{1}{u_{1}}+\frac{q}{u_{2}}+\frac{1}{2} x^{2}\left(1-\mu^{2}\right)(1+q)-\frac{1}{2} q\left(2+\frac{q}{1+q}\right)
$$


can be used as a geometry function, with

$$
\begin{aligned}
& u_{1}^{2}=x^{2}+\frac{2 q}{1+q} x \cos \varphi \sin \vartheta+\frac{q^{2}}{(1+q)^{2}}, \\
& u_{2}^{2}=x^{2}-\frac{2}{1+q} x \cos \varphi \sin \vartheta+\frac{1}{(1+q)^{2}}, \\
& q=M_{2} / M_{1}, \quad x=r / d
\end{aligned}
$$

where for the angular velocity

$$
\omega^{2}=G\left(M_{1}+M_{2}\right) / d^{3}
$$

is taken according to Kepler's law ( $d=$ distance between the two components). The origin of the system of polar coordinates $r, \vartheta, \varphi$ is in the center of mass of the binary system.

In principle the geometry function can be adjusted to the problem to be solved. If, for instance, the angular velocity is much higher near the axis of rotation, the equipotentials will have a stronger oblateness for $x$ in the neighborhood of zero, an effect which in principle can be taken into account by adding to the geometry function a term which represents the potential of a quadrupole-like symmetry.

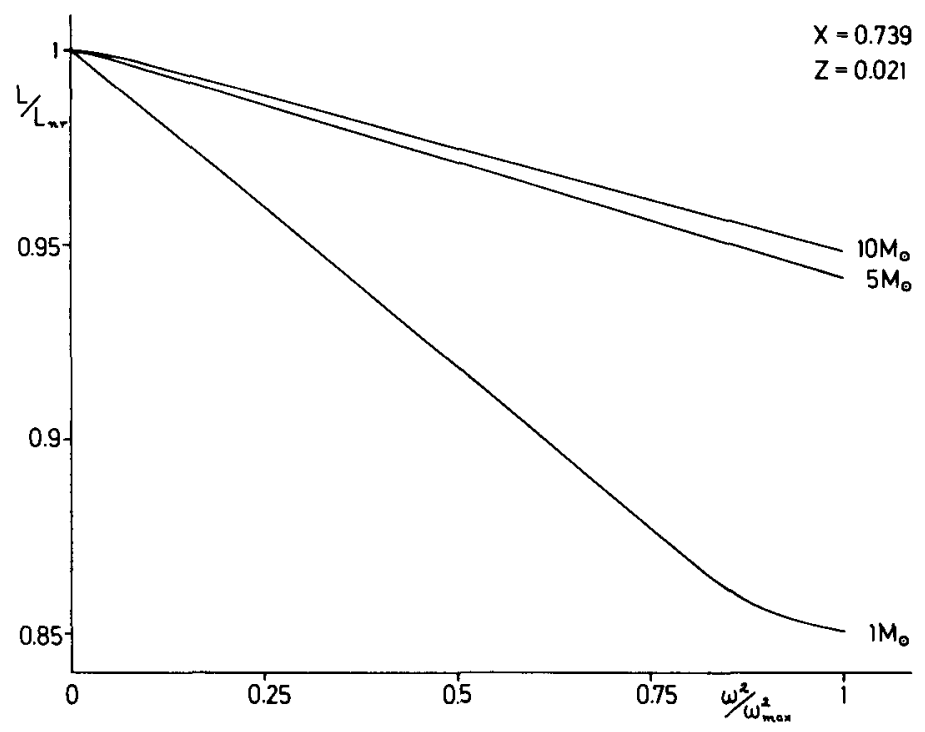

Fig. 2. Luminosity as a function of $\omega^{2}$ for uniformly rotating zero age main sequence stars of 1,5 , and $10 M_{\odot}$ with a chemical composition of $X=0.739, Z=0.021$. The units used are: maximum angular velocities $\omega_{\max }$ for the abscissa, and luminosities of the nonrotating models $L_{\mathrm{nr}}$ for the ordinate. The values of these units are:

\begin{tabular}{llll} 
& $1 M_{\odot}$ & $5 M_{\odot}$ & $10 M_{\odot}$ \\
\cline { 2 - 4 }$\omega_{\max } / \mathrm{sec}^{-1}$ & $5.124 \times 10^{-4}$ & $2.635 \times 10^{-4}$ & $1.493 \times 10^{-4}$ \\
$\log L_{\mathrm{n} r} / L_{\odot}$ & -0.1204 & 2.6558 & 3.6724
\end{tabular}




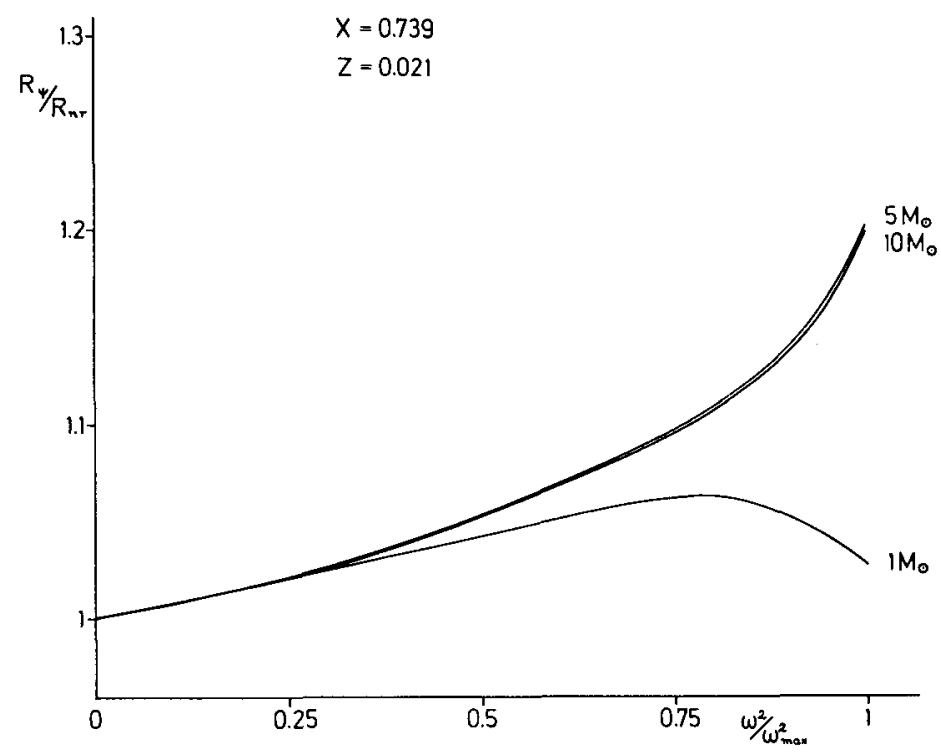

Fig. 3. Mean radius $R \Psi$ as a function of $\omega^{2}$ for uniformly rotating zero age main sequence stars of 1,5 , and $10 M_{\odot}$. Units for the abscissa are the same as in Figure 2; for the ordinate the radii $R_{\mathrm{nr}}$ of the nonrotating models were used. The values of these radii are

$\begin{array}{llll} & 1 M_{\odot} & 5 M_{\odot} & 10 M_{\odot} \\ R_{\mathrm{nr}} / \mathrm{cm} & 6.320 \times 10^{10} & 1.767 \times 10^{11} & 2.655 \times 10^{11}\end{array}$

One can imagine an iterative procedure, starting with a given geometry function, in which one solves the stellar structure equations and uses the density distribution obtained to improve the geometry function via the Poisson equation.

\section{Applications}

A. UNIFORMLY ROTATING MAIN SEQUENCE MODELS

Sets of models of different angular velocities have been computed for 1,5 , and $10 M_{\odot}$ using the geometry function (24). The tabulated correction factors were built into a normal stellar structure code based on the Henyey method. Starting with nonrotating models only a few iterations in the Henyey method were necessary to obtain the rotating models, even in the case of models rotating with the maximum possible angular velocity. Figures 2 and 3 give the results. The results agree well with those obtained by Faulkner et al. (1968) and by Sackmann and Anand (1969); our values are only slightly smaller. The only remarkable difference is the change in the mean radius for the $1 M_{\odot}$-model.

\section{B. UNIFORMLY ROTATING HELIUM MAIN SEQUENCE STARS}

For 1, 5, and $10 M_{\odot}$, helium stars, on the helium main sequence, have been computed for different angular velocities. The results are given in Figures 4 and 5 . 


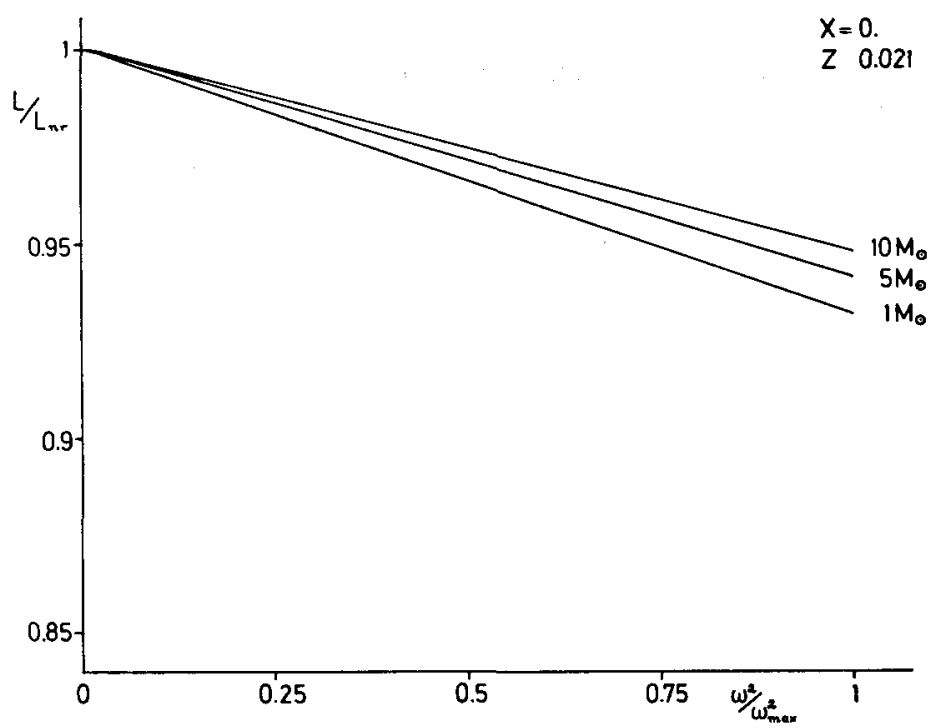

Fig. 4. Luminosity as a function of $\omega^{2}$ for uniformly rotating helium stars on the helium main sequence with masses 1,5 , and $10 M_{\odot}$ and a chemical composition of $Y=0.979, Z=0.021$. Units are defined as in Figure 2, their values being:

\begin{tabular}{llll} 
& $1 M_{\odot}$ & $5 M_{\odot}$ & $10 M_{\odot}$ \\
\cline { 2 - 4 }$\omega_{\max } / \mathrm{sec}^{-1}$ & $4.106 \times 10^{-2}$ & $1.699 \times 10^{-2}$ & $1.268 \times 10^{-2}$ \\
$\log L_{\mathrm{nr}} / L_{\odot}$ & 2.3727 & 4.4647 & 5.1374
\end{tabular}

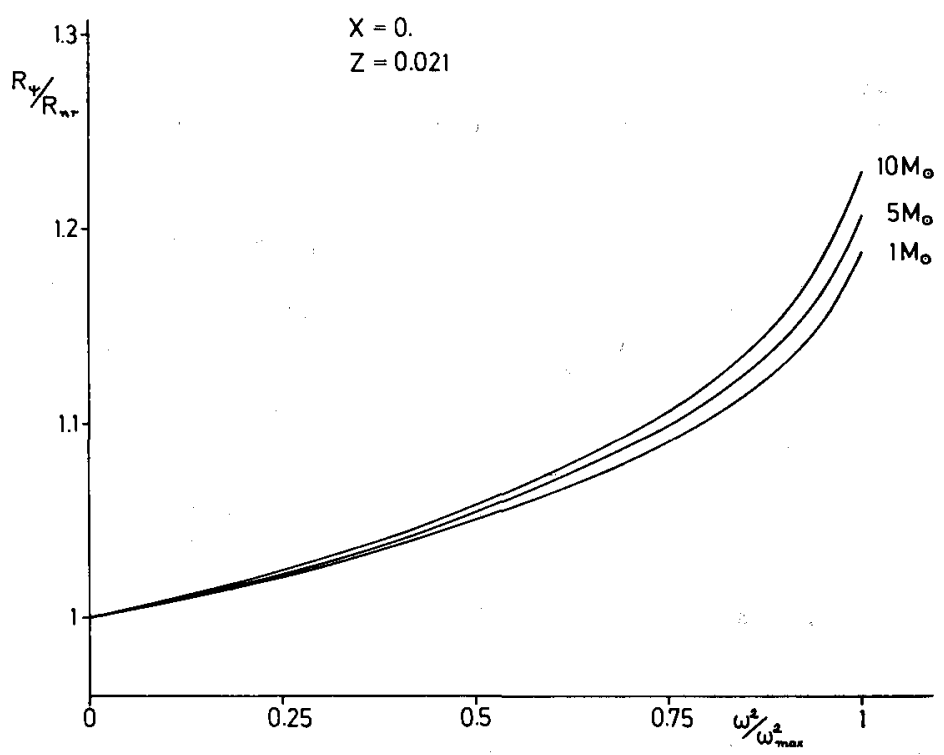

Fig. 5. Mean radius $R_{\boldsymbol{\Psi}}$ as a function of $\omega^{2}$ for uniformly rotating helium stars on the helium main sequence with masses 1,5 , and $10 M_{\odot}$. Units are defined as above, their values being:

$$
\begin{array}{llll}
R_{\mathrm{nr} / \mathrm{cm}} & 1 M_{\odot} & 5 M_{\odot} & 10 M_{\odot} \\
\hline 1.363 \times 10^{10} & 4.136 \times 10^{10} & 6.219 \times 10^{10}
\end{array}
$$


TABLE I

Comparison of a nonrotating and a fully rotating cooling white dwarf of the same central temperature

\begin{tabular}{llllll}
\hline $\log L / L_{\odot}$ & $L / L_{\mathrm{nr}}$ & $\log R_{\Psi}$ & $R_{\Psi} / R_{\mathrm{nr}}$ & $\log P_{\mathrm{c}}$ & $\log T_{\mathrm{c}}$ \\
\hline-2.4285 & 1 & 9.2099 & 1 & 21.6534 & 7.0371 \\
-2.6110 & 0.657 & 9.2794 & 1.174 & 21.5761 & 7.0371 \\
\hline
\end{tabular}

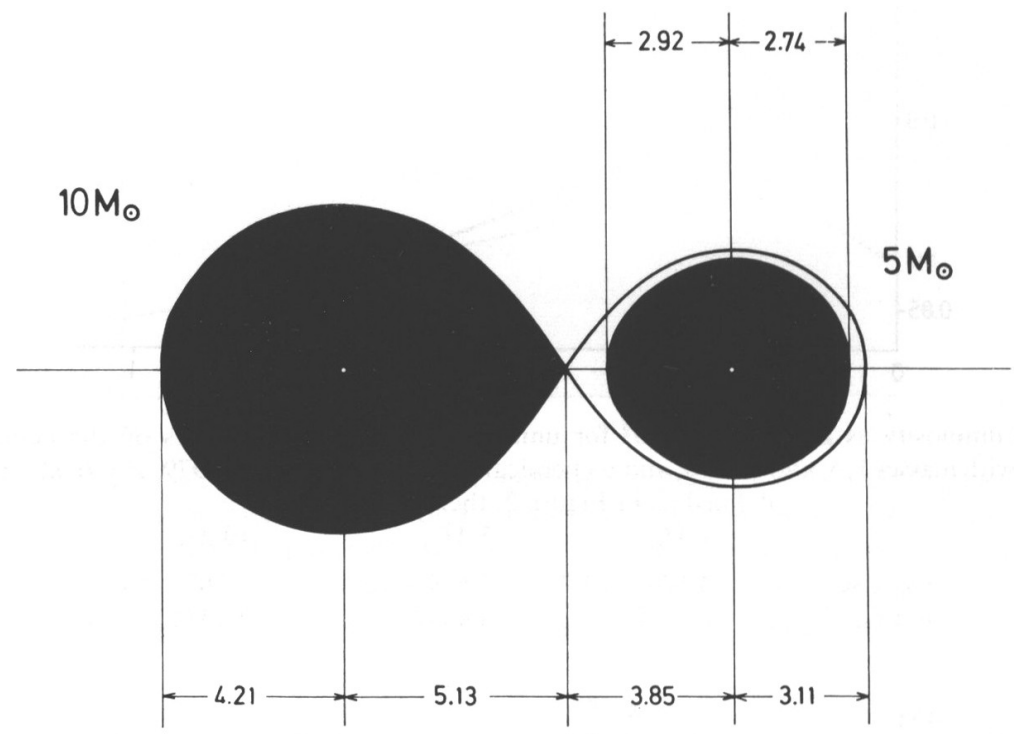

Fig. 6. The close binary system of $10+5 M_{\odot}$ in the plane of symmetry vertical to the rotation axis. Both stars are in the zero age stage. The numbers give distances in solar radii.

TABLE II

A zero age main sequence star of $10 M_{\odot}(X=0.739, Z=0.021)$ filling its critical lobe in a close binary system of $10+5 M_{\odot}$. For comparison, results for a nonrotating star of $10 M_{\odot}$ (line 2), a star rigidly rotating with the same angular velocity as the primary of the binary system (line 3 ), and a star rigidly rotating with the maximum angular velocity possible (line 4) are given. The value for $\omega_{\max }=$ $1.493 \times 10^{-4} \mathrm{sec}^{-1}$.

\begin{tabular}{lllll}
\hline$\omega^{2} / \omega^{2} \max$ & $\log L / L_{\odot}$ & $L / L_{\mathrm{nr}}$ & $\log R_{\psi}$ & $R_{\psi} / R_{\mathrm{nr}}$ \\
\hline 0.3655 & 3.6632 & 0.979 & 11.4414 & 1.041 \\
0 & 3.6724 & 1 & 11.4240 & 1 \\
0.3655 & 3.6632 & 0.979 & 11.4392 & 1.036 \\
1 & 3.6463 & 0.942 & 11.5028 & 1.200 \\
\hline
\end{tabular}

\section{A FULLY ROTATING WHITE DWARF MODEL}

A nonrotating white dwarf model of $0.23 M_{\odot}$ consisting of a helium core with an inactive hydrogen rich envelope has been taken from the computations of Kippenhahn et al. (1968) and compared with a fully rotating white dwarf model of the same central 
temperature and the same chemical structure. The latter again was computed with the method described above. Table I gives the comparisons.

\section{A COROTATING CLOSE BINARY SYSTEM}

The method has been applied to a system of $10+5 M_{\odot}$. The distance has been chosen in such a way that in the zero age stage the primary just fills its critical lobe (Figure 6). For both models the geometry function (32) has been used. While the secondary is only slightly distorted the primary shows the maximum effects due to tidal distortion. Table II gives the comparison between a spherical model for the primary, the nonspherical model derived by our method, and the model of a rotating star, rotating with the angular velocity of the binary system. One can see that within an accuracy of $10^{-4}$ the change in luminosity is caused by the rotation of the corotating binary system while the effect of the tidal forces can only be seen in a somewhat larger increase of the mean radius compared with the purely rotating stellar model. Similar conclusions were reached by Jackson (1970), who extended the method of Faulkner, Roxburgh, and Strittmatter by including tidal effects from a companion in a close binary system.

\section{References}

Faulkner, J., Roxburgh, I. W., and Strittmatter, P. A.: 1968, Astrophys. J. 151, 203.

Jackson, S.: 1970, Astrophys. J. (in press).

Kippenhahn, R., Thomas, H.-C., and Weigert, A.: 1968, Z. Astrophys. 69, 265.

Sackmann, I. J. and Anand, S. P. S.: 1969, preprint.

\section{Discussion}

Jordahl: Please specify more fully the variation in angular velocity with radius and why it was chosen.

Thomas: It was chosen such that $\omega^{2}=\omega_{\mathrm{s}}^{2} \cdot\left(M_{\mathrm{r}} / M\right), \omega_{\mathrm{s}}$ being the angular velocity at the surface, in order to simplify the geometry function. $\omega$ starts decreasing only when $f_{\mathrm{p}}$ and $f_{T}$ are already close to 1 , so the error will be negligible.

Ostriker: Can the change in sign you found (for the one solar mass model) in the quantity $\mathrm{d} r_{\psi} /$ $\mathrm{d}\left(\omega^{2}\right)$ be due to a change from primarily CNO cycle to PP chain as the central temperature decreases with increasing rotation?

Thomas: No, because in our $1 M \odot$ model the contribution of the CNO-cycle to nuclear energy production is small, even in the spherical model. 